\title{
Magnetic Particle Imaging Using Second-Harmonic Response of Magnetization
}

\author{
Tomoya Oishi*, Toshihumi Suzuki, Zhang $\mathrm{Yi}^{1}$ and Saburo Tanaka \\ Department of Environmental and Life Sciences, Toyohashi University of Technology \\ 1-1 Hibarigaoka, Tempaku-cho, Toyohashi, Aichi 441-8580, Japan \\ ${ }^{1}$ Peter Gruenberg Institute Forschungszentrum Juelich Juelich, D-52425, Germany
}

(Received February 24, 2015; accepted May 26, 2015)

Key words: magnetic particle imaging, MPI, magnetic nanoparticle, second harmonic, superparamagnetic

Magnetic particle imaging (MPI) is a new imaging modality that is capable of imaging the distribution of magnetic nanoparticles (MNPs) with high sensitivity, high spatial resolution, and high imaging speed. We applied an excitation magnetic field with frequency $\omega_{0}$ and an additional dc bias $H_{\mathrm{dc}}$ to obtain a second-harmonic response of magnetization $M$ generated owing to the nonlinearity of $M-H$. When $H_{\mathrm{dc}}$ is set at a knee point in the $M-H$ characteristics, the second harmonic reaches a maximum. This method does not need a large ac modulation field $H_{\mathrm{ac}}$. In this study, we developed a twodimensional (2-D) imaging system using the detection of the second-harmonic response of MNPs. As a result, we successfully obtained the one-dimensional (1-D) image of a phantom 60 -nm-diameter nanoparticle in a $\phi 2 \times 4 \mathrm{~mm}$ vessel. In the 1-D imaging map, the full width at half maximum (FWHM) was about $2 \mathrm{~mm}$, which was consistent with the phantom size $(\phi 2 \mathrm{~mm})$. In the $20 \times 8 \mathrm{~mm}^{2} 2$-D imaging map, the position of the two MNP phantoms could be identified.

\section{Introduction}

Magnetic nanoparticles (MNPs) have been well known as magnetic markers for biomedical applications. They are coated with a polymer, which is sometimes bound with diagnostic reagents or drugs on its surface. Magnetic markers have been used for the separation and purification of cells or pathogens and the amplification of contrast in MRI. Several magnetic detection methods have been developed to determine MNP volumes for use in different bioapplications, such as immunoassays. ${ }^{(1)}$

Magnetic particle imaging (MPI), as introduced by Gleich and Weizenecker, and Knopp et al., is based on utilizing the nonlinear magnetization response $M$ for the detection of superparamagnetic iron oxide nanoparticles. ${ }^{(2,3)}$ The spatial resolution of 1

${ }^{*}$ Corresponding author: e-mail: t135401@edu.tut.ac.jp 
mm can be obtained.(4) The sensitivity of MPI is one million times higher than that of magnetic resonance imaging (MRI). ${ }^{(4)}$

MPI is characterized by detecting the third harmonic of the magnetic response by applying an excitation ac magnetic field to MNPs. In MNPs detection and the MPI technique, the most commonly employed method is the detection of the odd harmonics of the $M$ response. We developed a method to improve the detection sensitivity for the magnetization $M$ of MNPs and an imaging technique based on the detection of the second-harmonic response. ${ }^{(5-7)}$ The advantage of using the second-harmonic response is that the response can be detected for even small amplitudes of the ac magnetic field. In the case of conventional detection using a third harmonic, the amplitude of the ac magnetic field must be larger than a threshold level.

In this study, we demonstrated the operation of a 2-D imaging system. A $20 \times 8 \mathrm{~mm}^{2}$ 2-D imaging map of two phantoms decorated with $60 \mathrm{~nm}$ diameter nanoparticles (Resovist ${ }^{\mathbb{R}}$ : Fujifilm RI Pharma Co., Ltd.) in a $\phi 2 \times 4 \mathrm{~mm}$ vessel was successfully generated. Phantom is a specially designed object scanned in the field of medical imaging and used for tuning the device.

\section{Principles of MNP Detection}

\subsection{Detection of $M N P S$}

MPI is a new technique that uses the nonlinear magnetization characteristics of MNPs to generate images. The MNP (particle diameter $\leq 100 \mathrm{~nm}$ ) used for MPI has no hysteresis in the magnetization curve as contrasted with ferromagnetic materials such as Fe and Co. Therefore, an MNP has no residual magnetization, and the magnitude of magnetization is determined uniquely by the magnetic field strength.

Figure 1(a) shows how to detect the third-harmonic response of the MNP. An ac magnetic field is applied at the origin of the $M-H$ curve of the MNP. If an ac magnetic field with smaller amplitude $\left(H_{\mathrm{acl}}\right)$ is applied, the $M$ response becomes sinusoidal and contains the fundamental frequency $\left(M_{1}\right)$. However, in the case of a large ac magnetic

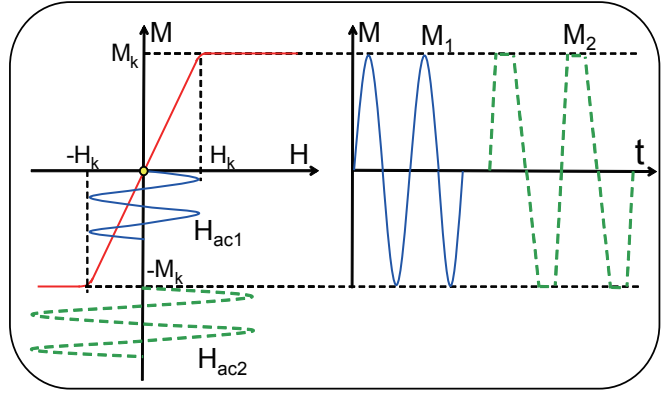

(a)

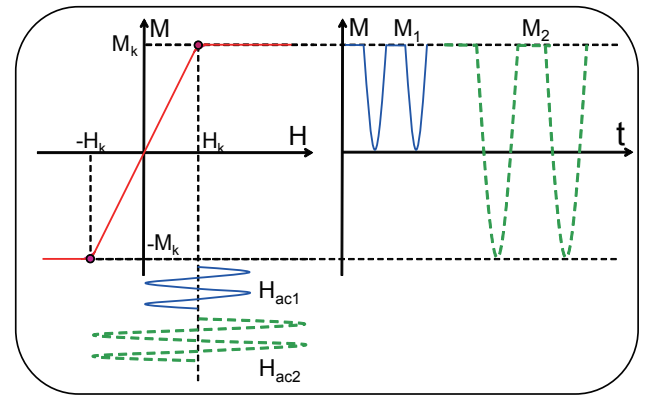

(b)

Fig. 1. (Color online) Principle of MNP detection using harmonic response: (a) using the third harmonic and (b) using the second harmonic. 
field $\left(H_{\mathrm{ac} 2}\right)$, the $M$ response is distorted and contains the third and odd harmonics $\left(M_{2}\right)$. This indicates that an amplitude larger than that of the saturation magnetization $H_{\mathrm{k}}$ of the $M-H$ curve of the MNP is essential in principle to obtain the third-harmonic response. It is expected that a large power source and a large coil will be required.

Figure 1(b) shows how to detect the second-harmonic response of the MNP. When an ac magnetic field $\left(H_{\mathrm{ac}}\right)$ and an additional dc bias field $H_{\mathrm{dc}}$ are applied, the $M$ response is a half-rectified waveform and contains primarily the second harmonic. Since the $M-H$ curve follows the Langevin function, the characteristics are not simple and round at the bias field $H_{\mathrm{dc}}=H_{\mathrm{k}}$. However, when the dc bias field $H_{\mathrm{dc}}=H_{\mathrm{k}}$, the second harmonic of $M$ reaches a maximum owing to the nonlinearity of the $M-H$ characteristics. The waveform of $M$ is not changed by the amplitude of the ac magnetic field $\left(H_{\mathrm{ac} 1}\right.$ or $\left.H_{\mathrm{ac} 2}\right)$. The advantage of using the second-harmonic response is that the response can be observed for even small amplitudes of $H_{\mathrm{ac}}$. Usually, an amplitude of $10 \mathrm{mT} / \mu_{0}$ should be applied, but almost half of it is applied in our experiment. ${ }^{(2)}$

\subsection{Principles of 1-D and 2-D imaging processes using second harmonic}

In this section, we explain how to carry out 1-D and 2-D imaging processes for MNPs using the second harmonic of the magnetic response.

A magnetic field gradient is required for MPI. To realize a 1-D MPI system, we placed a pair of $\mathrm{NdFeB}$ permanent magnets at both ends of a particle detection system. The magnets generated a gradient field transverse to the imaging bore. The two saturation points, $H_{\mathrm{k}}$, occur side-by-side on the $M-H$ curve, which gives a maximum second-harmonic response, as shown in Fig. 1(b). To realize 1-D imaging, the saturation points $H_{\mathrm{k}}$ must be scanned. The principle is shown in Fig. 2. By changing the dc bias field, the gradient field line can be scanned from left (open circle) to right (solid circle), and the response from an object in the imaging bore can be detected.

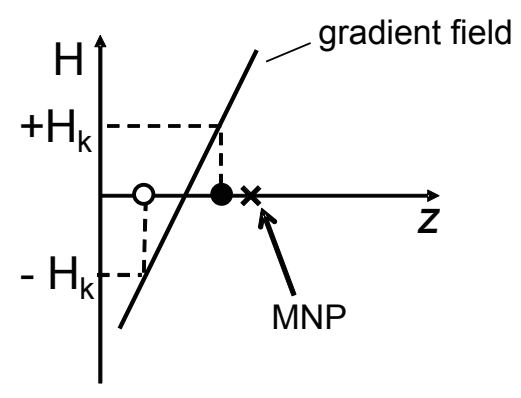

(a)

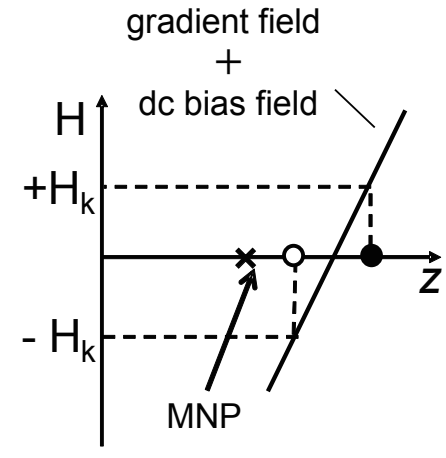

(b)

Fig. 2. Scanning of $H_{\mathrm{k}}$ by applying a dc bias field: (a) before scanning and (b) after scanning. 
If an MNP is in the imaging region, the signal of the second-harmonic peak appears twice. These two peaks correspond to $-H_{\mathrm{k}}$ and $+H_{\mathrm{k}}$, and the position of the phantom is the middle between these peaks. To identify the position, a lock-in amplifier was introduced into the MPI scanning system. Since the phase between two peaks $\left(-H_{\mathrm{k}}\right.$ and $\left.+H_{\mathrm{k}}\right)$ is 180 degrees, if the second-harmonic peaks are detected by a lock-in amplifier, the signals can be converted into the shape shown in Fig. 3(a). Moreover, the partial differentiation of the cosine component $d(\cos \theta) / d z$ can be calculated to clearly identify the position of the phantom as shown in Fig. 3(b).

Figure 4 shows a schematic of the 2-D imaging system using MNPs. This 2-D imaging system is fundamentally the same as the 1-D imaging system except for an $x y$ positioner. The phantom was scanned along the $z$-axis by applying a dc bias field. After scanning along the $z$-axis with an applied dc bias field, the position of the phantom was moved in the $x$-axis by the $x y$-positioner. By repeating the scan, it was possible to obtain an $x z$ 2-D image of the phantom.

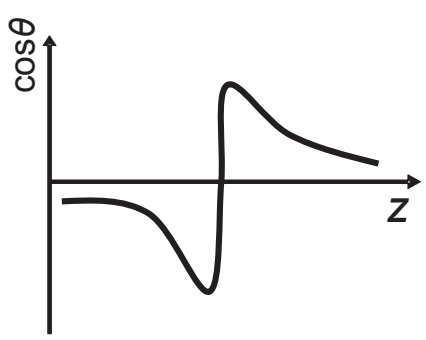

(a)

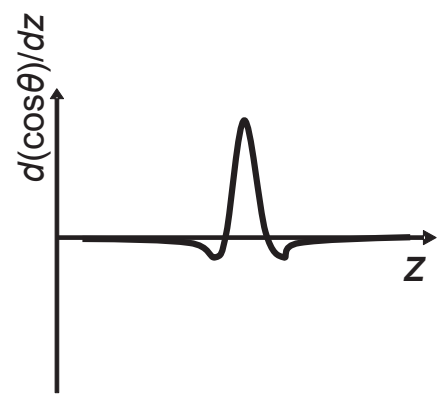

(b)

Fig. 3. Principle of imaging MNPs using a phase-sensitive detector: (a) $\cos \theta \operatorname{component}$ and (b) $d(\cos \theta) / d z$.

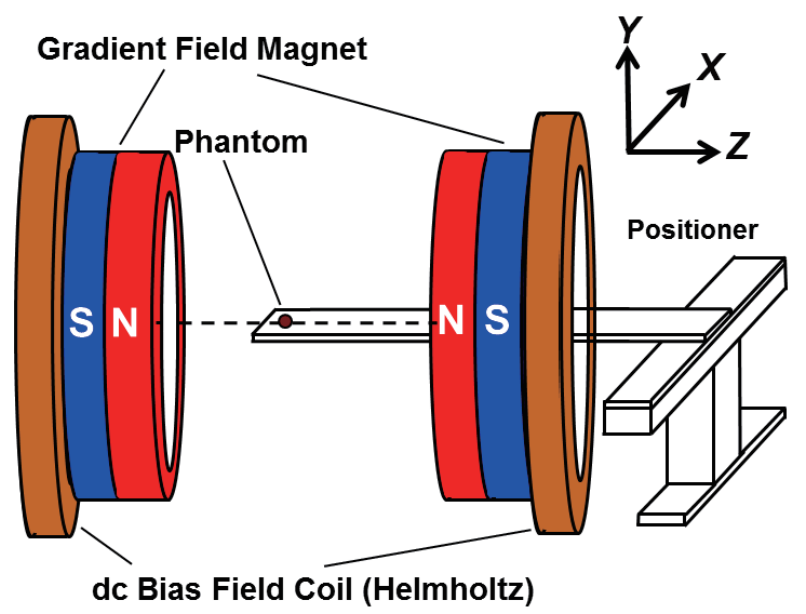

Fig. 4. (Color online) Schematic of the 2-D imaging system using MNPs. 


\section{MPI Scanning System}

\subsection{Setup for 2-D imaging system}

Figure 5 shows a schematic diagram of the MPI scanning system. The MPI scanning system was constructed with a permanent ring magnet, a dc bias field coil (Helmholtz), an ac modulation field coil, and a differential detection coil. The MPI scanning system used a pair of $\mathrm{NdFeB}$ permanent ring magnets with a bore size of $\phi 80 \mathrm{~mm}$ separated by $90 \mathrm{~mm}$. The magnets generated gradients of $G_{z}=3.18 \mathrm{~T} / \mathrm{m}$ transverse to the imaging bore and $G_{x}=1.26 \mathrm{~T} / \mathrm{m}$ along the longitudinal axis.

The ac modulation field and dc bias field coils were used to generate an excitation field $H_{\mathrm{ac}}$ and a static field $H_{\mathrm{dc}}$, respectively. The detection coil, which consisted of two differential connected coils, detected the magnetic response of the MNP sample.

The ac modulation field and detection coils were arranged coaxially. The coil parameters, including the measured resistance and impedance, are listed in Table 1. The specifications of the MNPs are listed in Table 2. In the experiments, the amplitude of the static field generated by the dc bias field coil reached values up to $\pm 32 \mathrm{mT} / \mu_{0}$. An ac modulation field up to $11 \mathrm{mT}_{\mathrm{p}-\mathrm{p}} / \mu_{0}$ at $2 \mathrm{kHz}$ (fundamental frequency) was applied. The sample rod with a small vessel $(\phi 2 \times 4 \mathrm{~mm})$ was located on one side of the detection coil.

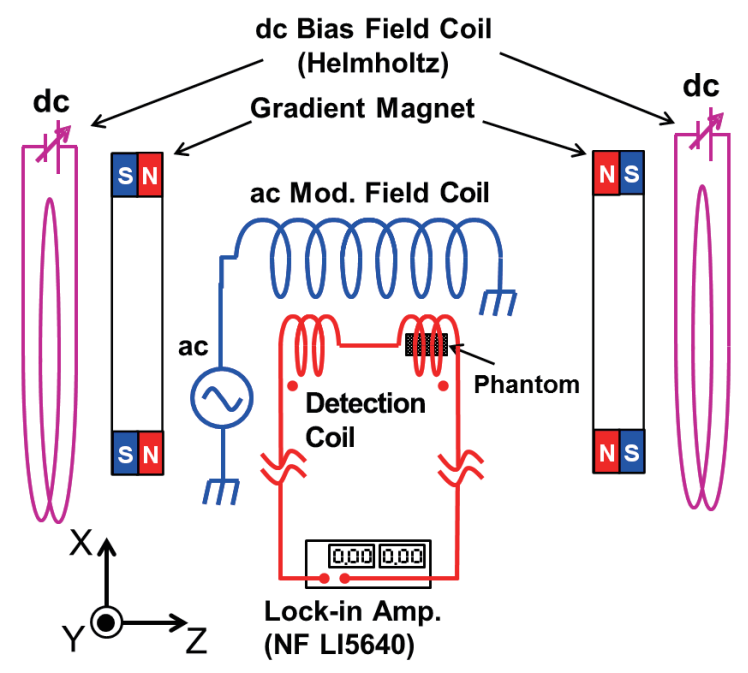

Fig. 5. (Color online) Schematic diagram of the MPI scanning system. The scanning system was constructed with a permanent ring magnet, a dc bias field coil (Helmholtz), an ac modulation field coil, and a differential detection coil. A lock-in amplifier was used to detect the second-harmonic response of the phantom. 
Table 1

Coil parameters.

\begin{tabular}{lccccc}
\hline & $\begin{array}{c}\text { I.D. } \times \text { Length } \\
(\mathrm{mm})\end{array}$ & $\begin{array}{c}\text { Wire size } \\
(\mathrm{mm})\end{array}$ & No. of turns & $\begin{array}{c}\text { Resistance } \\
(\Omega)\end{array}$ & $\begin{array}{c}\text { Inductance } \\
(\mathrm{mH})\end{array}$ \\
\hline dc bias field coil & $\phi 90 \times(94+94)$ & $1.0 \times 3.0$ & $1485 \times 2$ & $5.5 /$ coil & $132 /$ coil \\
ac modulation field coil & $\phi 40 \times 260$ & $\phi 0.6$ & 744 & 6.1 & 3.8 \\
Detection coil & $\phi 17 \times(50+50)$ & $\phi 0.6$ & $1000 \times 2$ & 12.4 & 12.9 \\
\hline
\end{tabular}

Table 2

MNP parameters.

\begin{tabular}{lccc}
\hline Name & Core & Fe content $(\mathrm{mg} / \mathrm{ml})$ & Particle diameter $(\mathrm{nm})$ \\
\hline Resovist $^{\circledR 8}$ & $\mathrm{Fe}_{2} \mathrm{O}_{3}$ & 27.8 & $45-65$ \\
\hline
\end{tabular}

\section{$3.2 \quad 1-D$ imaging and results}

An original Resovist ${ }^{\circledR}$ solution $(10 \mu \mathrm{l})$ was used as a sample. An ac modulation field amplitude of $5.5 \mathrm{mT} / \mu_{0}$ at $2 \mathrm{kHz}$ was applied, and the dc bias field in the $z$-axis was scanned within the range of $\pm 32 \mathrm{mT} / \mu_{0}$, which corresponded to $\pm 10 \mathrm{~mm}$. The step in the $z$-axis was $\pm 0.13 \mathrm{mT} / \mu_{0}(0.04 \mathrm{~mm}$ intervals $)$. The second-harmonic response signal from the detection coil was amplified with a lock-in amplifier (LI5640, NF Corporation). The cosine component of the response at $4 \mathrm{kHz}$ in the lock-in amplifier was selected and recorded by a PC at each position. The 1-D image of the phantom, a nanoparticle solution $\left(\right.$ Resovist $^{\mathbb{R}}$ ) in a $\phi 2 \times 4 \mathrm{~mm}$ vessel, is shown in Fig. 6 . The black line shows the cosine component of the second-harmonic response, which indicates two peaks at a dc bias field of $\pm 3 \mathrm{mT} / \mu_{0}$. The intensity of the second-harmonic signal was about $0.7 \mathrm{mV}_{\mathrm{p}-\mathrm{p}}$. The red line shows the partial differentiation of the $\operatorname{cosine} d(\cos \theta) / d z$. The full width at half maximum (FWHM) was about $2 \mathrm{~mm}$, which was almost the same as the phantom diameter $(\phi 2 \mathrm{~mm})$.

\subsection{2-D imaging and results}

For 2-D imaging, we used two phantoms. Two phantoms were placed at a separation of $5 \mathrm{~mm}$ at the center of one side of the detection coil. A dc bias field in the $z$-axis was scanned within the range of $\pm 32 \mathrm{mT} / \mu_{0}$, which corresponds to $\pm 10 \mathrm{~mm}$. After scanning along the $z$-axis, the samples were moved in the $x$-axis by a positioner by a step of 1 $\mathrm{mm}$. This protocol was then repeated until areas of $\pm 4 \mathrm{~mm}$ in the $x$-axis and $\pm 10 \mathrm{~mm}$ in the $z$-axis were scanned. A 2-D imaging map of the two phantoms, 60 -nm-diameter nanoparticles in solution (Resovist ${ }^{\circledR}$ ) in $\phi 2 \times 4 \mathrm{~mm}$ vessels, is shown in Fig. 7. The cosine component of the response at each position is plotted in the $z x$ plate. However, since the gradient fields in the $z$ - and $x$-axes $\left(G_{z}\right.$ and $G_{x}$, respectively) had a 2.5 difference in magnitude, each signal in the $x$-axis was multiplied by a factor of $1 / 2.5$. Figure 7 (a) shows the cosine component of the second-harmonic response. Two magnetic dipoles 


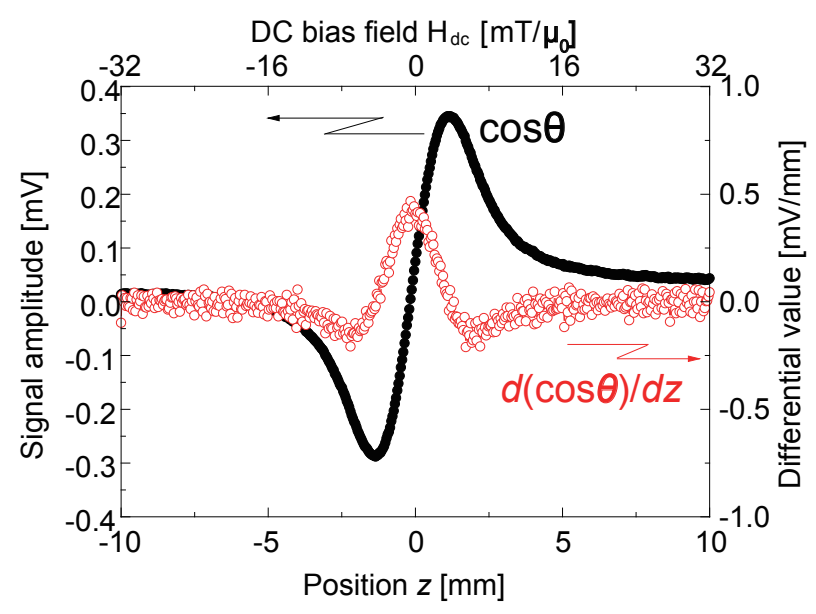

Fig. 6. (Color online) Cosine component (solid circles) and derivative values (open circles) plotted as a function of the position $z$ and de bias field.

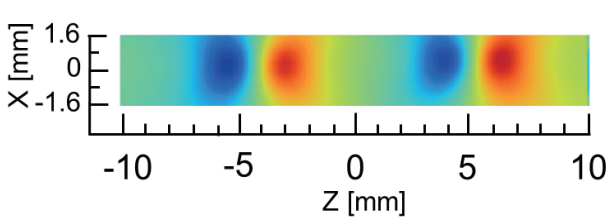

(a)

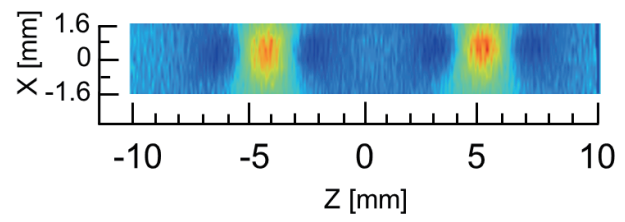

(b)

Fig. 7. (Color online) Reconstruction of 2-D imaging maps of two phantoms. Resovist ${ }^{\circledR}(10 \mu \mathrm{l})$ in $\phi 2 \times 4 \mathrm{~mm}$ vessels: (a) 2-D imaging map from the cosine component of the second-harmonic response and (b) 2-D imaging map from the partial differentiation of the $\operatorname{cosine} d(\cos \theta) / d z$.

were located at a separation of $5 \mathrm{~mm}$ along the $z$-axis. Figure 7(b) shows the partial differentiation of the $\operatorname{cosine} d(\cos \theta) / d z$. The position of the two phantoms could be identified at positions of $\pm 5 \mathrm{~mm}$ from the center.

\section{Conclusions}

We constructed a 2-D imaging scanning system and demonstrated 2-D imaging. Nanoparticle solutions of Resovist ${ }^{\mathbb{R}}$ were used as phantoms. The lock-in amp technique was employed to manipulate the cosine component to determine the position of the phantom. As a result, a 2-D dipole contour map of the two magnetic particle solutions was successfully generated. Moreover, the positions of the MNP solutions were clearly indicated in the contour map following the partial differentiation of the cosine component. 


\section{References}

1 H.-J. Krause, N. Wolters, Y. Zhang, A. Offenhaeussera, P. Miethe, M. H. F. Meyer, M. Hartmann and M. Keusgen: J. Magn. Magn. Mater. 311 (2007) 436.

2 B. Gleich and J. Weizenecker: Nature 435 (2005) 1214.

3 T. Knopp, S. Biederer, T. Sattel, J. Rahmer, J.Weizenecker, B. Gleich, J. Borgert and T. Buzug: Med. Phys. 37 (2010) 486.

4 P. W. Goodwill and S. M. Conolly: IEEE Trans. Med. Imaging 30 (2011) 1581.

5 J. B. Weaver, A. M. Rauwerdink, C. R. Sullivan and I. Baker: Med. Phys. 35 (2008) 1988.

6 T. Yoshida, K. Ogawa, T. Tsubaki, N. B. Othman and K. Enpuku: IEEE Trans. Magn. 47 (2011) 2863.

7 Y. Zhang, H. Murata, Y. Hatsukade and S. Tanaka: Rev. Sci. Instrum. 84 (2013) 094702. 